\title{
Periodic solutions for second order integro-differential equations with infinite delay in Banach spaces
}

\author{
by \\ Shangquan Bu and Yi FAng (Beijing)
}

\begin{abstract}
We study the maximal regularity on different function spaces of the second order integro-differential equations with infinite delay

(P) $\quad u^{\prime \prime}(t)+\alpha u^{\prime}(t)+\frac{d}{d t}\left(\int_{-\infty}^{t} b(t-s) u(s) d s\right)=A u(t)-\int_{-\infty}^{t} a(t-s) A u(s) d s+f(t)$

$(0 \leq t \leq 2 \pi)$ with periodic boundary conditions $u(0)=u(2 \pi), u^{\prime}(0)=u^{\prime}(2 \pi)$, where $A$ is a closed operator in a Banach space $X, \alpha \in \mathbb{C}$, and $a, b \in L^{1}\left(\mathbb{R}_{+}\right)$. We use Fourier multipliers to characterize maximal regularity for $(P)$. Using known results on Fourier multipliers, we find suitable conditions on the kernels $a$ and $b$ under which necessary and sufficient conditions are given for the problem $(P)$ to have maximal regularity on $L^{p}(\mathbb{T}, X)$, periodic Besov spaces $B_{p, q}^{s}(\mathbb{T}, X)$ and periodic Triebel-Lizorkin spaces $F_{p, q}^{s}(\mathbb{T}, X)$.
\end{abstract}

1. Introduction. In a series of recent publications operator-valued Fourier multipliers on vector-valued function spaces have been studied (see e.g. $[2,3,1,6,14,15])$. They are needed to study the existence and uniqueness of differential equations on Banach spaces. In $[2,3,1,6]$, the authors study the maximal regularity of the classical second order problem $\left(P_{1}\right)$ on $L^{p}$ spaces, Besov spaces and Triebel-Lizorkin spaces using operator-valued Fourier multipliers, where

$$
\left(P_{1}\right) \quad\left\{\begin{array}{l}
u^{\prime \prime}(t)+A u(t)=f(t) \quad(0 \leq t \leq 2 \pi) \\
u(0)=u(2 \pi), \quad u^{\prime}(0)=u^{\prime}(2 \pi)
\end{array}\right.
$$

here $A$ is a closed linear operator defined in a Banach space $X$ and $f$ is an $X$-valued function defined on $[0,2 \pi]$. If $X$ is a UMD Banach space and

2000 Mathematics Subject Classification: Primary 45N05; Secondary 45D05, 43A15, $47 \mathrm{D} 99$.

Key words and phrases: Fourier multiplier, maximal regularity, integro-differential equation, Besov spaces, Triebel-Lizorkin spaces.

This work was supported by the NSF of China (10571099), Specialized Research Fund for the Doctoral Program of Higher Education and the Tsinghua Basic Research Foundation (JCpy2005056). 
$1<p<\infty$, then the problem $\left(P_{1}\right)$ has maximal regularity on $L^{p}(\mathbb{T}, X)$ if and only if $k^{2} \in \varrho(A)$ for all $k \in \mathbb{Z}$ and the sequence $\left(k^{2} R\left(k^{2}, A\right)\right)_{k \in \mathbb{Z}}$ is Rademacher bounded [2]. In the setting of Besov spaces $B_{p, q}^{s}(\mathbb{T}, X)$ and Triebel-Lizorkin spaces $F_{p, q}^{s}(\mathbb{T}, X)$, the maximal regularity is equivalent to the condition that $k^{2} \in \varrho(A)$ for all $k \in \mathbb{Z}$ and $\left(k^{2} R\left(k^{2}, A\right)\right)_{k \in \mathbb{Z}}$ is bounded $[3,6]$.

In this paper, we consider a more general evolution equation, namely the second order integro-differential equation with infinite delay:

$$
\left\{\begin{aligned}
u^{\prime \prime}(t) & +B u^{\prime}(t)+\frac{d}{d t}\left(\int_{-\infty}^{t} b(t-s) u(s) d s\right) \\
& =A u(t)-\int_{-\infty}^{t} a(t-s) A u(s) d s+f(t) \quad(0 \leq t \leq 2 \pi), \\
u(0)=u(2 \pi), \quad u^{\prime}(0)=u^{\prime}(2 \pi), &
\end{aligned}\right.
$$

where $A$ and $B$ are closed linear operators in a Banach space $X$ and $a, b \in$ $L^{1}\left(\mathbb{R}_{+}\right)$. Much literature has been devoted to a similar first order integrodifferential equation $\left(P_{3}\right)$ :

$$
\left(P_{3}\right)\left\{\begin{array}{l}
\gamma_{0} u^{\prime}(t)+\frac{d}{d t}\left(\int_{-\infty}^{t} b(t-s) u(s) d s\right)+\gamma_{\infty} u(t) \\
=c_{0} A u(t)-\int_{-\infty}^{t} a(t-s) A u(s) d s+f(t) \quad(0 \leq t \leq 2 \pi), \\
u(0)=u(2 \pi),
\end{array}\right.
$$

where $\gamma_{0}, \gamma_{\infty}, c_{0}$ are constants, $A$ is a closed linear operator in $X$, and $a, b \in L^{1}\left(\mathbb{R}_{+}\right)$. The class of equations of type $\left(P_{2}\right)$ and $\left(P_{3}\right)$ arises as models for nonlinear heat conduction in materials of fading memory type, and in population dynamics. In [11], Keyantuo and Lizama obtained the maximal regularity of $\left(P_{3}\right)$ on $L^{p}$ spaces and Besov spaces. They also studied this equation in the case $\gamma_{0}=c_{0}=1, b=\gamma_{\infty}=0$ in a previous paper [10]. Clément and Da Prato [8] studied $\left(P_{3}\right)$ on the real line in the case $a=0$ and obtained maximal regularity results in Sobolev spaces and Hölder spaces as well as in the space of bounded uniformly continuous functions. Da Prato and Lunardi [9] investigated periodic solutions of $\left(P_{3}\right)$ in the case $b=0$. Hölder continuous solutions of $\left(P_{3}\right)$ have been studied on the real line by Lunardi [12] in the case of $A$ being the Laplacian operator in a bounded domain $\Omega \subset \mathbb{R}^{N}$ and $X=C(\bar{\Omega})$.

We notice that the problem $\left(P_{2}\right)$ has been studied by several authors in a simpler form and for different boundary conditions. For instance, R. Chill 
and S. Srivastava [7] have considered the $L^{p}$-maximal regularity on a finite interval $[0, T)$ for the abstract second order problem

$$
\left(P_{4}\right)\left\{\begin{array}{l}
u^{\prime \prime}(t)+B u^{\prime}(t)+A u(t)=f(t) \quad(0 \leq t<T), \\
u(0)=0, \quad u^{\prime}(0)=0 .
\end{array}\right.
$$

The semigroup theory and trace spaces played important roles in that discussion. Under a suitable condition on the operators $A$ and $B$, they gave a necessary and sufficient condition for the problem $\left(P_{4}\right)$ to have $L^{p}$-maximal regularity.

In this paper, we are interested in the second order integro-differential equation $\left(P_{2}\right)$ with periodic boundary conditions. Since $A$ and $B$ are not necessarily generators of semigroups in our situation, semigroup theory is no longer applicable. So our main tool in the study of maximal regularity of $\left(P_{2}\right)$ is operator-valued Fourier multipliers. The presence of two closed linear operators in the operator-valued multiplier functions makes the verification of the sufficient condition for Fourier multipliers particularly complicated. Therefore in this paper, we just consider the simpler case $B=\alpha I$ for some fixed $\alpha \in \mathbb{C}$ (the general case will be studied elsewhere).

We want to obtain maximal regularity of $\left(P_{2}\right)$ with $B=\alpha I$ for some $\alpha \in \mathbb{C}$ on three function spaces: $L^{p}(\mathbb{T}, X)$ for $1<p<\infty$, periodic Besov spaces $B_{p, q}^{s}(\mathbb{T}, X)$ for $1 \leq p, q \leq \infty, s>0$, and periodic Triebel-Lizorkin spaces $F_{p, q}^{s}(\mathbb{T}, X)$ for $1 \leq p<\infty, 1 \leq q \leq \infty, s>0$, where $\mathbb{T}=[0,2 \pi]$. The main tools are the operator-valued Fourier multiplier theorems obtained in [2, Theorem 1.3], [3, Theorem 4.5] and [6, Theorem 3.2]. The differences between these multiplier theorems on different function spaces make us impose different conditions on the kernels $a$ and $b$ to obtain the maximal regularity on these spaces. These conditions are satisfied by a class of functions which correspond to the most common kernels encountered in applications. Furthermore, it is easy to see that in the case $\alpha=0, a=b=0$ our results are in accordance with the well known results for $\left(P_{1}\right)[2,3,6]$.

The paper is organized as follows. In Section 2, we establish a general maximal regularity result for a problem $\left(P_{2}\right)$ in the case $B=\alpha I$ for some $\alpha \in \mathbb{C}$, in terms of operator-valued Fourier multipliers. In Section 3 , we apply the general result to three concrete function spaces: $L^{p}(\mathbb{T}, X), B_{p, q}^{s}(\mathbb{T}, X)$ and $F_{p, q}^{s}(\mathbb{T}, X)$, still in the case $B=\alpha I$ for some $\alpha \in \mathbb{C}$.

2. Maximal regularity via Fourier multipliers. Let $X$ be a Banach space. We will consider the problem $\left(P_{2}\right)$ in a simpler form 


$$
\left(P_{5}\right)\left\{\begin{aligned}
u^{\prime \prime}(t) & +\alpha u^{\prime}(t)+\frac{d}{d t}\left(\int_{-\infty}^{t} b(t-s) u(s) d s\right) \\
& =A u(t)-\int_{-\infty}^{t} a(t-s) A u(s) d s+f(t) \quad(0 \leq t \leq 2 \pi), \\
u(0) & =u(2 \pi), \quad u^{\prime}(0)=u^{\prime}(2 \pi),
\end{aligned}\right.
$$

where $A$ is a closed linear operator in $X, a, b \in L^{1}\left(\mathbb{R}_{+}\right), f$ is an $X$-valued function defined on $\mathbb{T}:=[0,2 \pi]$ and $\alpha \in \mathbb{C}$ is a constant. The solution of $\left(P_{5}\right)$ will be an $X$-valued function defined on $\mathbb{T}$ (extended to $\mathbb{R}$ by periodicity).

Fourier multipliers will be very useful in our study of maximal regularity of the problem $\left(P_{5}\right)$ on different function spaces. These spaces include $L^{p}(\mathbb{T}, X)$ for $1<p<\infty, B_{p, q}^{s}(\mathbb{T}, X)$ for $1 \leq p, q \leq \infty, s>0$ and $F_{p, q}^{s}(\mathbb{T}, X)$ for $1 \leq p<\infty, 1 \leq q \leq \infty, s>0$. For detailed information about vectorvalued periodic Besov and Triebel-Lizorkin spaces, we refer to [3, Section 2] and $[6$, Section 2].

If $Y$ is another Banach space, we denote by $\mathcal{L}(X, Y)$ the space of all bounded linear operators from $X$ to $Y$. If $X=Y$, we will simply denote it by $\mathcal{L}(X)$. For $1 \leq p \leq \infty$ and $f \in L^{p}(\mathbb{T}, X)$, we denote by

$$
\widehat{f}(k)=\frac{1}{2 \pi} \int_{0}^{2 \pi} e_{-k}(t) f(t) d t
$$

the $k$ th Fourier coefficient of $f$, where $k \in \mathbb{Z}$ and $e_{k}(t)=e^{i k t}$ for $t \in \mathbb{R}$. For $x \in X$, we let $e_{k} \otimes x$ be the $X$-valued function given by $t \mapsto e_{k}(t) x$.

Definition 2.1. Let $X$ and $Y$ be Banach spaces and let $\Gamma(\mathbb{T}, X)$ be one of the following $X$-valued function spaces: $L^{p}(\mathbb{T}, X)(1 \leq p<\infty), B_{p, q}^{s}(\mathbb{T}, X)$ $(1 \leq p, q \leq \infty, s \in \mathbb{R})$ or $F_{p, q}^{s}(\mathbb{T}, X)(1 \leq p<\infty, 1 \leq q \leq \infty, s \in \mathbb{R})$. We say that a sequence $\left(M_{k}\right)_{k \in \mathbb{Z}} \subset \mathcal{L}(X, Y)$ is a $\Gamma$-multiplier if for each $f \in \Gamma(\mathbb{T}, X)$, there exists a unique $g \in \Gamma(\mathbb{T}, Y)$ such that $\widehat{g}(k)=M_{k} \widehat{f}(k)$ for all $k \in \mathbb{Z}$ $[2,3,6]$.

REMARK 2.2. 1. It follows from the closed graph theorem that if $\left(M_{k}\right)_{k \in \mathbb{Z}} \subset \mathcal{L}(X, Y)$ is a $\Gamma$-multiplier, then there exists a constant $C>0$ such that for $f \in \Gamma(\mathbb{T}, X)$, we have $\left\|\sum_{k \in \mathbb{Z}} e_{k} \otimes M_{k} \widehat{f}(k)\right\|_{\Gamma} \leq C\|f\|_{\Gamma}$. This implies that each $\Gamma$-multiplier is a bounded sequence.

2. It is clear from the definition that if $\left(M_{k}\right)_{k \in \mathbb{Z}} \subset \mathcal{L}(X, Y)$ and $\left(N_{k}\right)_{k \in \mathbb{Z}}$ $\subset \mathcal{L}(Y, Z)$ are $\Gamma$-multipliers, then so is $\left(N_{k} M_{k}\right)_{k \in \mathbb{Z}} \subset \mathcal{L}(X, Z)$.

Let $X$ be a Banach space and let $\Gamma(\mathbb{T}, X)$ be one of the following: $L^{p}(\mathbb{T}, X)(1<p<\infty), B_{p, q}^{s}(\mathbb{T}, X)(1 \leq p, q \leq \infty, s>0)$ or $F_{p, q}^{s}(\mathbb{T}, X)$ $(1 \leq p<\infty, 1 \leq q \leq \infty, s>0)$. We denote the first order "Sobolev" space by $\Gamma^{[1]}(\mathbb{T}, X)$ and the second order "Sobolev" spaces by $\Gamma^{[2]}(\mathbb{T}, X)$ : 
if $\Gamma(\mathbb{T}, X)=L^{p}(\mathbb{T}, X)$, then

$$
\begin{array}{r}
\Gamma^{[1]}(\mathbb{T}, X)=\left\{u \in L^{p}(\mathbb{T}, X): \text { there exists } v \in L^{p}(\mathbb{T}, X)\right. \\
\text { such that } \widehat{v}(k)=i k \widehat{u}(k) \text { for all } k \in \mathbb{Z}\} \\
=\left\{u \in L^{p}(\mathbb{T}, X): u \text { is differentiable a.e., } u^{\prime} \in L^{p}(\mathbb{T}, X),\right. \\
\text { and } u(0)=u(2 \pi)\} .
\end{array}
$$

$\Gamma^{[2]}(\mathbb{T}, X)=\left\{u \in L^{p}(\mathbb{T}, X):\right.$ there exists $v \in L^{p}(\mathbb{T}, X)$

$$
\text { such that } \left.\widehat{v}(k)=-k^{2} \widehat{u}(k) \text { for all } k \in \mathbb{Z}\right\} \text {, }
$$

$=\left\{u \in L^{p}(\mathbb{T}, X): u\right.$ is twice differentiable a.e., $u^{\prime}, u^{\prime \prime} \in L^{p}(\mathbb{T}, X)$,

$$
\text { and } \left.u(0)=u(2 \pi), u^{\prime}(0)=u^{\prime}(2 \pi)\right\} \text {. }
$$

If $\Gamma(\mathbb{T}, X)=B_{p, q}^{s}(\mathbb{T}, X)(1 \leq p, q \leq \infty, s>0)$, then

$$
\Gamma^{[1]}(\mathbb{T}, X)=B_{p, q}^{s+1}(\mathbb{T}, X), \quad \Gamma^{[2]}(\mathbb{T}, X)=B_{p, q}^{s+2}(\mathbb{T}, X) .
$$

If $\Gamma(\mathbb{T}, X)=F_{p, q}^{s}(\mathbb{T}, X)(1 \leq p<\infty, 1 \leq q \leq \infty, s>0)$, then

$$
\Gamma^{[1]}(\mathbb{T}, X)=F_{p, q}^{s+1}(\mathbb{T}, X), \quad \Gamma^{[2]}(\mathbb{T}, X)=F_{p, q}^{s+2}(\mathbb{T}, X) .
$$

We refer to [2, Section 2, 6], [3, Section 2] and [6, Section 2] for more information about these spaces. For $g \in L^{1}\left(\mathbb{R}_{+}\right)$and $u \in L^{1}(\mathbb{T}, X)$ (extended to $\mathbb{R}$ by periodicity), we define

$$
F(t)=(g \dot{*} u)(t):=\int_{-\infty}^{t} g(t-s) u(s) d s .
$$

In this notation, $\left(P_{5}\right)$ has the following more compact form:

$$
u^{\prime \prime}+\alpha u^{\prime}+\frac{d}{d t}(b \dot{*} u)=A u-a \dot{*} u+f
$$

with periodic boundary conditions $u(0)=u(2 \pi), u^{\prime}(0)=u^{\prime}(2 \pi)$.

Let $\widetilde{g}(\lambda)=\int_{0}^{\infty} e^{-\lambda t} g(t) d t$ be the Laplace transform of $g$. An easy computation shows that

$$
\widehat{F}(k)=\widetilde{g}(i k) \widehat{u}(k) \quad(k \in \mathbb{Z}) .
$$

Now we define the $\Gamma$-maximal regularity of the problem $\left(P_{5}\right)$.

Definition 2.3. Let $X$ be a Banach space, $A$ be a closed linear operator in $X, \alpha \in \mathbb{C}$ and let $a, b \in L^{1}\left(\mathbb{R}_{+}\right)$. Let $\Gamma(\mathbb{T}, X)$ be one of the following: $L^{p}(\mathbb{T}, X)(1<p<\infty), B_{p, q}^{s}(\mathbb{T}, X)(1 \leq p, q \leq \infty, s>0)$ or $F_{p, q}^{s}(\mathbb{T}, X)$ $(1 \leq p<\infty, 1 \leq q \leq \infty, s>0)$,

1. Let $f \in \Gamma(\mathbb{T}, X)$. A function $u \in \Gamma^{[2]}(\mathbb{T}, X)$ is called a strong $\Gamma$ solution of $\left(P_{5}\right)$ if $u(t) \in D(A)$ and the equation of $\left(P_{5}\right)$ holds for almost all $t \in \mathbb{T}$, and $u^{\prime \prime}, u^{\prime}, A u, a \dot{*} A u, \frac{d}{d t}(b \dot{*} u) \in \Gamma(\mathbb{T}, X)$. 
2. The problem $\left(P_{5}\right)$ is said to have $\Gamma$-maximal regularity if for every $f \in \Gamma(\mathbb{T}, X)$, there exists a unique strong $\Gamma$-solution of $\left(P_{5}\right)$.

In what follows, we always set $g_{k}=\widetilde{g}(i k)$ for any $g \in L^{1}\left(\mathbb{R}_{+}\right)$and $R(\lambda, A)=(\lambda-A)^{-1}$ for $\lambda \in \varrho(A)$, where $\varrho(A)$ is the resolvent set of $A$. If $a \in \mathbb{C}$, we will simply denote the bounded linear operator $a I$ by $a$, where $I$ is the identity of $X$. We consider the following two hypotheses for a scalar function $g$ defined on $\mathbb{R}_{+}$:

(H0a) $g \in L^{1}\left(\mathbb{R}_{+}\right)$and $\left(g_{k}\right)_{k \in \mathbb{Z}} \subset \mathcal{L}(X)$ is a $\Gamma$-multiplier.

(HOb) $g_{k} \neq 1$ for all $k \in \mathbb{Z}$ and $\left(\left(1-g_{k}\right)^{-1}\right)_{k \in \mathbb{Z}} \subset \mathcal{L}(X)$ is a $\Gamma$-multiplier.

We shall write (HO) when (HOa) and (HOb) are both satisfied. For convenience, for $a, b \in L^{1}\left(\mathbb{R}_{+}\right)$we adopt the following notations: for $k \in \mathbb{Z}$,

$$
\begin{aligned}
a_{k} & :=\widetilde{a}(i k), \quad b_{k}:=\widetilde{b}(i k), \\
d_{k} & :=\frac{i k\left(\alpha+b_{k}\right)-k^{2}}{1-a_{k}}, \\
M_{k} & :=\frac{-k^{2}}{1-a_{k}} R\left(d_{k}, A\right) .
\end{aligned}
$$

Now, we are ready to state the main result of this section.

Theorem 2.4. Let $X$ be a Banach space, $A: D(A) \subset X \rightarrow X$ be a closed linear operator, $\alpha \in \mathbb{C}$ and let $a, b \in L^{1}\left(\mathbb{R}_{+}\right)$. Let $\Gamma(\mathbb{T}, X)$ be one of the following: $L^{p}(\mathbb{T}, X)(1<p<\infty), B_{p, q}^{s}(\mathbb{T}, X)(1 \leq p, q \leq \infty, s>0)$ or $F_{p, q}^{s}(\mathbb{T}, X)(1 \leq p<\infty, 1 \leq q \leq \infty, s>0)$. Assume that a satisfies (H0) and $b$ satisfies (H0a). Then the following assertions are equivalent:

(i) The problem $\left(P_{5}\right)$ has $\Gamma$-maximal regularity.

(ii) $\left(d_{k}\right)_{k \in \mathbb{Z}} \subset \varrho(A)$ and $\left(M_{k}\right)_{k \in \mathbb{Z}}$ is a $\Gamma$-multiplier.

Proof. We notice that if $s>0$, then $B_{p, q}^{s}(\mathbb{T}, X)$ and $F_{p, q}^{s}(\mathbb{T}, X)$ embed continuously into $L^{p}(\mathbb{T}, X)[3,6]$, thus we will freely use results in $L^{p}(\mathbb{T}, X)$ for functions in $B_{p, q}^{s}(\mathbb{T}, X)$ or $F_{p, q}^{s}(\mathbb{T}, X)$ when $s>0$.

(i) $\Rightarrow$ (ii): Let $k \in \mathbb{Z}$ and $y \in X$ be fixed. We define $f(t)=e^{i k t} y$. Then $\widehat{f}(k)=y$. By assumption, there exists $u \in \Gamma^{[2]}(\mathbb{T}, X)$ such that $u(t) \in D(A)$ and

$$
u^{\prime \prime}(t)+\alpha u^{\prime}(t)+\frac{d}{d t}(b \dot{*} u(t))=A u(t)-a \dot{*} A u(t)+f(t)
$$

for almost all $t \in \mathbb{T}, u^{\prime \prime}, u^{\prime}, A u, a \dot{*} A u, \frac{d}{d t}(b \dot{*} u) \in \Gamma(\mathbb{T}, X)$ and $u(0)=$ $u(2 \pi), u^{\prime}(0)=u^{\prime}(2 \pi)$. Taking Fourier transforms on both sides, using (2.2) and the closedness of $A$, we find that $\widehat{u}(k) \in D(A)$ and

$$
\left[-k^{2}+i k \alpha+i k b_{k}-\left(1-a_{k}\right) A\right] \widehat{u}(k)=y .
$$

Thus $-k^{2}+i k \alpha+i k b_{k}-\left(1-a_{k}\right) A$ is surjective. To show that it is also injective, let $x \in D(A)$ be such that $\left[-k^{2}+i k \alpha+i k b_{k}-\left(1-a_{k}\right) A\right] x=0$. 
Then

$$
A x=\frac{-k^{2}+i k\left(\alpha+b_{k}\right)}{1-a_{k}} x=d_{k} x,
$$

where we have used the assumption that the kernel $a$ satisfies (H0) and therefore $a_{k}-1 \neq 0$ for $k \in \mathbb{Z}$. Hence $u(t)=e^{i k t} x$ defines a solution of $u^{\prime \prime}(t)+\alpha u^{\prime}(t)+\frac{d}{d t}(b \dot{*} u(t))=A u(t)-a \dot{*} A u(t), u(0)=u(2 \pi), u^{\prime}(0)=u^{\prime}(2 \pi)$. Indeed,

$$
\begin{aligned}
A u(t) & -\int_{-\infty}^{t} a(t-s) A u(s) d s=A e^{i k t} x-\int_{-\infty}^{t} a(t-s) A e^{i k s} x d s \\
& =e^{i k t} A x-e^{i k t} a_{k} A x=\left(1-a_{k}\right) e^{i k t} A x=\left[-k^{2}+i k\left(\alpha+b_{k}\right)\right] e^{i k t} x \\
& =u^{\prime \prime}(t)+\alpha u^{\prime}(t)+\frac{d}{d t}(b \dot{*} u(t)) .
\end{aligned}
$$

By the uniqueness assumption, we have $x=0$. We have shown that $-k^{2}+$ $i k \alpha+i k b_{k}-\left(1-a_{k}\right) A$ is bijective. Since $A$ is closed, we conclude that

$$
d_{k}=\frac{-k^{2}+i k \alpha+i k b_{k}}{1-a_{k}} \in \varrho(A) \quad \text { for each } k \in \mathbb{Z} .
$$

Next, we show that $\left(M_{k}\right)_{k \in \mathbb{Z}}$ is a $\Gamma$-multiplier where $M_{k}$ is defined by (2.3). If $f \in \Gamma(\mathbb{T}, X)$, there exists $u \in \Gamma^{[2]}(\mathbb{T}, X)$ solving $\left(P_{5}\right)$ by assumption. Taking Fourier transforms, we obtain

$$
\left[-k^{2}+i k \alpha+i k b_{k}-\left(1-a_{k}\right) A\right] \widehat{u}(k)=\widehat{f}(k) \quad(k \in \mathbb{Z}) .
$$

Since $-k^{2}+i k \alpha+i k b_{k}-\left(1-a_{k}\right) A$ is invertible, we have

$$
\widehat{u}(k)=\frac{1}{1-a_{k}} R\left(d_{k}, A\right) \widehat{f}(k) \quad \text { and } \quad-k^{2} \widehat{u}(k)=M_{k} \widehat{f}(k) .
$$

Since $u \in \Gamma^{[2]}(\mathbb{T}, X)$, it is twice differentiable a.e. on $\mathbb{T}, u^{\prime}, u^{\prime \prime} \in \Gamma(\mathbb{T}, X)$ and

$$
\widehat{u^{\prime \prime}}(k)=-k^{2} \widehat{u}(k)=M_{k} \widehat{f}(k) \quad(k \in \mathbb{Z}) .
$$

From this and the definition of $\Gamma$-multiplier, we conclude that $\left(M_{k}\right)_{k \in \mathbb{Z}}$ is a $\Gamma$-multiplier.

(ii) $\Rightarrow(\mathrm{i})$ : Let $f \in \Gamma(\mathbb{T}, X)$. We define

$$
N_{k}=\frac{1}{1-a_{k}} R\left(d_{k}, A\right) \text {. }
$$

By Remark 2.2, $N_{k}=\left(-1 / k^{2}\right) M_{k}$ and $i k N_{k}=(1 / i k) M_{k}$ are $\Gamma$-multipliers as the sequences $\left(-1 / k^{2}\right)_{k \in \mathbb{Z}}$ and $(1 / i k)_{k \in \mathbb{Z}}$ are $\Gamma$-multipliers by $[2$, Theorem 1.3], [3, Theorem 4.5] and [6, Theorem 3.2]. Since $\left(N_{k}\right)_{k \in \mathbb{Z}}$ is a $\Gamma$-multiplier, there exists $u \in \Gamma(\mathbb{T}, X)$ such that $\widehat{u}(k)=N_{k} \widehat{f}(k)$ for all $k \in \mathbb{Z}$. This implies that $\widehat{u}(k) \in D(A)$ and

$$
\left[-k^{2}+i k\left(\alpha+b_{k}\right)-\left(1-a_{k}\right) A\right] \widehat{u}(k)=\widehat{f}(k)
$$


for all $k \in \mathbb{Z}$. Since $\left(i k N_{k}\right)_{k \in \mathbb{Z}}$ is also a $\Gamma$-multiplier, there exists $v \in \Gamma(\mathbb{T}, X)$ such that

$$
\widehat{v}(k)=i k N_{k} \widehat{f}(k)=i k \widehat{u}(k) .
$$

By [2, Lemma 2.1], $u$ is differentiable a.e. with $v=u^{\prime}$ and $u(0)=u(2 \pi)$. Therefore $u \in \Gamma^{[1]}(\mathbb{T}, X)$. As $\left(M_{k}\right)_{k \in \mathbb{Z}}$ is a $\Gamma$-multiplier by assumption, there exists $w \in \Gamma(\mathbb{T}, X)$ such that

$$
\widehat{w}(k)=M_{k} \widehat{f}(k)=i k \widehat{v}(k)=-k^{2} \widehat{u}(k) .
$$

By [2, Lemma 2.1], $v=u^{\prime}$ is differentiable a.e. with $w=v^{\prime}=u^{\prime \prime}$ and $u^{\prime}(0)=u^{\prime}(2 \pi)$. This implies that $u \in \Gamma^{[2]}(\mathbb{T}, X)$.

Next, we show that $u(t) \in D(A)$ for almost all $t \in \mathbb{T}$. We have remarked that for $k \in \mathbb{Z}$, we have $\widehat{u}(k) \in D(A)$ and

$$
\begin{aligned}
A \widehat{u}(k) & =\frac{-k^{2} \widehat{u}(k)}{1-a_{k}}+\frac{\left(\alpha+b_{k}\right) i k \widehat{u}(k)}{1-a_{k}}-\frac{\widehat{f}(k)}{1-a_{k}} \\
& =\frac{\widehat{w}(k)}{1-a_{k}}+\frac{\left(\alpha+b_{k}\right) \widehat{v}(k)}{1-a_{k}}-\frac{\widehat{f}(k)}{1-a_{k}} .
\end{aligned}
$$

In view of assumptions (H0) on $a$ and (HOa) on $b$ and the facts that $w, v, f \in$ $\Gamma(\mathbb{T}, X) \subset L^{1}(\mathbb{T}, X)$, there exists $g \in \Gamma(\mathbb{T}, X)$ such that $A \widehat{u}(k)=\widehat{g}(k)$. Then by [2, Lemma 3.1], $u(t) \in D(A)$ for almost all $t \in \mathbb{T}$ and $A u \in \Gamma(\mathbb{T}, X)$. Clearly,

$$
\left(\frac{d}{d t}(b \dot{*} u)\right)^{\wedge}(k)=i k b_{k} \widehat{u}(k)=b_{k}\left(i k N_{k}\right) \widehat{f}(k)
$$

and

$$
(a \dot{*} A u)^{\wedge}(k)=a_{k} A \widehat{u}(k)=a_{k} \widehat{g}(k) .
$$

Since $\left(a_{k}\right)_{k \in \mathbb{Z}},\left(b_{k}\right)_{k \in \mathbb{Z}}$ and $\left(i k N_{k}\right)_{k \in \mathbb{Z}}$ are $\Gamma$-multipliers, we conclude that $\frac{d}{d t}(b \dot{*} u), a \dot{*} A u \in \Gamma(\mathbb{T}, X)$.

Now, from (2.4) and the uniqueness theorem of Fourier coefficients, we conclude that $u(t)$ satisfies $\left(P_{5}\right)$ for a.e. $t \in[0,2 \pi]$. This shows the existence.

To show the uniqueness, let $u \in \Gamma^{[2]}(\mathbb{T}, X)$ be such that

$$
u^{\prime \prime}(t)+\alpha u^{\prime}(t)+\frac{d}{d t}(b \dot{*} u(t))-A u(t)+a \dot{*} A u(t)=0
$$

for almost all $t \in \mathbb{T}$ and $u(0)=u(2 \pi), u^{\prime}(0)=u^{\prime}(2 \pi)$. Then taking Fourier transforms we have $\widehat{u}(k) \in D(A)$ and $\left[-k^{2}+i k\left(\alpha+b_{k}\right)-\left(1-a_{k}\right) A\right] \widehat{u}(k)=0$ by $\left[2\right.$, Lemma 3.1]. Since $d_{k}=\left(-k^{2}+i k\left(\alpha+b_{k}\right)\right) /\left(1-a_{k}\right) \in \varrho(A)$, we must have $\widehat{u}(k)=0$ for all $k \in \mathbb{Z}$. Thus $u=0$ and the proof is finished.

We remark that on a Hilbert space $X$, each bounded sequence is an $L^{2}$ multiplier. By the Riemann-Lebesgue lemma, if $a \in L^{1}\left(\mathbb{R}_{+}\right)$, then $\lim _{k \rightarrow \infty} a_{k}$ $=0$. Thus on a Hilbert space $X$ the above theorem takes a particularly simple form: 
Corollary 2.5. Let $X$ be a Hilbert space, $A: D(A) \subset X \rightarrow X$ be a closed linear operator, $\alpha \in \mathbb{C}$ and let $a, b \in L^{1}\left(\mathbb{R}_{+}\right)$. Assume that $a_{k} \neq 1$ for all $k \in \mathbb{Z}$. Then the following assertions are equivalent:

(i) The problem $\left(P_{5}\right)$ has $L^{2}$-maximal regularity.

(ii) $\left(d_{k}\right)_{k \in \mathbb{Z}} \subset \varrho(A)$ and $\sup _{k \in \mathbb{Z}}\left\|M_{k}\right\|<\infty$.

3. Maximal regularity on three function spaces. In this section, we apply Theorem 2.4 in three concrete function spaces: $L^{p}(\mathbb{T}, X)(1<$ $p<\infty), B_{p, q}^{s}(\mathbb{T}, X)(1 \leq p, q \leq \infty, s>0)$ and $F_{p, q}^{s}(\mathbb{T}, X)(1 \leq p<\infty$, $1 \leq q \leq \infty, s>0$ by imposing some conditions on the kernels $a, b \in L^{1}\left(\mathbb{R}_{+}\right)$. The three operator-valued multiplier theorems obtained in $[2,3,6]$ on these function spaces are fundamental for our discussion. Versions of the multiplier theorems on the real line can be found in $[14,15]$.

For results about R-boundedness, we can refer to Bourgain [4], Weis $[14,15]$ and Arendt-Bu [2]. We merely recall the definition and some basic properties.

We let $r_{j}$ be the $j$ th Rademacher function on $[0,1]$ given by $r_{j}(t)=$ $\operatorname{sgn}\left(\sin \left(2^{j-1} t\right)\right)$. For $x \in X$, we denote by $r_{j} \otimes x$ the vector-valued function $t \mapsto r_{j}(t) x$.

Definition 3.1. Let $X$ and $Y$ be Banach spaces. A family $\mathbf{T} \subset \mathcal{L}(X, Y)$ is called $R$-bounded if there exists $C \geq 0$ such that

$$
\left\|\sum_{j=1}^{n} r_{j} \otimes T_{j} x_{j}\right\|_{L^{1}(0,1 ; Y)} \leq C\left\|\sum_{j=1}^{n} r_{j} \otimes x_{j}\right\|_{L^{1}(0,1 ; X)}
$$

for all $T_{1}, \ldots, T_{n} \in \mathbf{T}, x_{1}, \ldots, x_{n} \in X$ and $n \in \mathbb{N}$.

REMARK 3.2 .

(a) Let $\mathbf{S}, \mathbf{T} \subset \mathcal{L}(X)$ be R-bounded sets. Then it is clear from the definition that $\mathbf{S T}:=\{S T: S \in \mathbf{S}, T \in \mathbf{T}\}$ is R-bounded.

(b) Each subset $\mathbf{M} \subset \mathcal{L}(X)$ of the form $\mathbf{M}=\{\lambda I: \lambda \in \Omega\}$ is R-bounded whenever $\Omega \subset \mathbb{C}$ is bounded. This follows from Kahane's contraction principle $[13, \S 3.5 .4]$.

In order to state our main results, we will use the following hypotheses for a scalar function $a \in L^{1}\left(\mathbb{R}_{+}\right)$(we recall that the sequence $\left(a_{k}\right)_{k \in \mathbb{Z}}$ is defined by (2.3)):

(H1a) $\left(k\left(a_{k+1}-a_{k}\right)\right)_{k \in \mathbb{Z}}$ is bounded.

(H1b) $a_{k} \neq 1$ for all $k \in \mathbb{Z}$.

(H2) $\left(k a_{k}\right)_{k \in \mathbb{Z}}$ and $\left(k^{2}\left(a_{k+1}-2 a_{k}+a_{k-1}\right)\right)_{k \in \mathbb{Z}}$ are bounded.

(H3) $\left(k a_{k}\right)_{k \in \mathbb{Z}},\left(k^{2}\left(a_{k+1}-2 a_{k}+a_{k-1}\right)\right)_{k \in \mathbb{Z}}$ and $\left(k^{3}\left(a_{k+1}-3 a_{k}+3 a_{k-1}\right.\right.$ $\left.\left.-a_{k-2}\right)\right)_{k \in \mathbb{Z}}$ are bounded. 
Remark 3.3. From [11, Remarks 3.4 and 3.5], we know that these conditions are satisfied by a large class of functions, which correspond to the most common kernels encountered in applications. When we refer simply to $(\mathbf{H} 1)$, we mean $(\mathbf{H 1} \mathbf{a})$ and $(\mathbf{H} 1 \mathbf{b})$.

LEMMA 3.4.

(1) Let $X$ be a UMD space. Assume that $a \in L^{1}\left(\mathbb{R}_{+}\right)$satisfies $(\mathbf{H 1})$ and $b \in L^{1}\left(\mathbb{R}_{+}\right)$satisfies $(\mathbf{H 1 a})$. Then $\left(a_{k}\right)_{k \in \mathbb{Z}},\left(\left(1-a_{k}\right)^{-1}\right)_{k \in \mathbb{Z}}$ and $\left(b_{k}\right)_{k \in \mathbb{Z}}$ are $L^{p}$-multipliers whenever $1<p<\infty$.

(2) Let $X$ be a Banach space. Assume that $a, b \in L^{1}\left(\mathbb{R}_{+}\right)$satisfy (H2) and a satisfies $(\mathbf{H 1} \mathbf{b})$. Then $\left(a_{k}\right)_{k \in \mathbb{Z}},\left(\left(1-a_{k}\right)^{-1}\right)_{k \in \mathbb{Z}}$ and $\left(b_{k}\right)_{k \in \mathbb{Z}}$ are $B_{p, q}^{s}$-multipliers whenever $1 \leq p, q \leq \infty$ and $s \in \mathbb{R}$.

(3) Let $X$ be a Banach space. Assume that $a, b \in L^{1}\left(\mathbb{R}_{+}\right)$satisfy $(\mathbf{H 3})$ and a satisfies $(\mathbf{H 1} \mathbf{b})$. Then $\left(a_{k}\right)_{k \in \mathbb{Z}},\left(\left(1-a_{k}\right)^{-1}\right)_{k \in \mathbb{Z}}$ and $\left(b_{k}\right)_{k \in \mathbb{Z}}$ are $F_{p, q}^{s}$-multipliers whenever $1 \leq p<\infty, 1 \leq q \leq \infty$ and $s \in \mathbb{R}$.

These assertions follow from [10, Lemmas 2.9 and 3.8] and [5, Proposition 3.4]. We omit the details. The following is one of the main results of this paper.

TheOREm 3.5. Let $X$ be a UMD space and let $A$ be a closed linear operator in $X$. Assume that $a, b \in L^{1}\left(\mathbb{R}_{+}\right)$satisfy $(\mathbf{H 1})$ and $(\mathbf{H 1} \mathbf{a})$, respectively. Then the following statements are equivalent:

(i) The problem $\left(P_{5}\right)$ has $L^{p}$-maximal regularity for some (equivalently, all) $1<p<\infty$.

(ii) $\left(d_{k}\right)_{k \in \mathbb{Z}} \subset \varrho(A)$ and $\left(M_{k}\right)_{k \in \mathbb{Z}}$ is $R$-bounded.

Proof. Since $a \in L^{1}\left(\mathbb{R}_{+}\right)$satisfies $(\mathbf{H 1})$ and $b \in L^{1}\left(\mathbb{R}_{+}\right)$satisfies $(\mathbf{H 1 a})$, it follows that $a$ satisfies (H0) and $b$ satisfies (H0a) by Lemma 3.4. Thus Theorem 2.4 is applicable in the case $\Gamma(\mathbb{T}, X)=L^{p}(\mathbb{T}, X)$ when $1<p<\infty$.

(i) $\Rightarrow$ (ii): Assume that $\left(P_{5}\right)$ has $L^{p}$-maximal regularity for some $1<p$ $<\infty$. By Theorem 2.4, $\left(d_{k}\right)_{k \in \mathbb{Z}} \subset \varrho(A)$ and $\left(M_{k}\right)_{k \in \mathbb{Z}}$ is an $L^{p}$-multiplier. The R-boundedness of $\left(M_{k}\right)_{k \in \mathbb{Z}}$ follows from [2, Proposition 1.11].

(ii) $\Rightarrow$ (i): Fix $1<p<\infty$, and assume that $\left(d_{k}\right)_{k \in \mathbb{Z}} \subset \varrho(A)$ and $\left(M_{k}\right)_{k \in \mathbb{Z}}$ is R-bounded. In view of Theorem 2.4, it suffices to show that $\left(M_{k}\right)_{k \in \mathbb{Z}}$ is an $L^{p}$-multiplier. We define

$$
\mu_{k}=k^{2} R\left(d_{k}, A\right)=-\left(1-a_{k}\right) M_{k} .
$$

Then $\mu_{k}$ is R-bounded by Remark 3.2. We claim that $\left(k\left(\mu_{k+1}-\mu_{k}\right)\right)_{k \in \mathbb{Z}}$ is also R-bounded. Indeed,

$$
\begin{aligned}
k\left(\mu_{k+1}-\mu_{k}\right) & =k\left[(k+1)^{2} R\left(d_{k+1}, A\right)-k^{2} R\left(d_{k}, A\right)\right] \\
& =k\left[(k+1)^{2}\left(R\left(d_{k+1}, A\right)-R\left(d_{k}, A\right)\right)+(2 k+1) R\left(d_{k}, A\right)\right]
\end{aligned}
$$




$$
\begin{aligned}
& =k(k+1)^{2}\left(d_{k}-d_{k+1}\right) R\left(d_{k+1}, A\right) R\left(d_{k}, A\right)+(2 k+1) k R\left(d_{k}, A\right) \\
& =\frac{d_{k}-d_{k+1}}{k} \mu_{k} \mu_{k+1}+\frac{2 k+1}{k} \mu_{k} .
\end{aligned}
$$

We have

$$
\begin{aligned}
\frac{d_{k}-d_{k+1}}{k}= & \frac{i\left(\alpha+b_{k}\right)}{1-a_{k}}-\frac{k+1}{k} \frac{i\left(\alpha+b_{k+1}\right)}{1-a_{k+1}} \\
& +\frac{2 k+1}{k} \frac{1}{1-a_{k+1}}+\frac{k\left(a_{k+1}-a_{k}\right)}{\left(1-a_{k}\right)\left(1-a_{k+1}\right)},
\end{aligned}
$$

which is clearly bounded. From the assumption on $a, b \in L^{1}\left(\mathbb{R}_{+}\right)$and Lemma 3.4, we know that $\left(\left(d_{k}-d_{k+1}\right) / k\right)_{k \in \mathbb{Z}}$ is R-bounded. It follows that $\left(k\left(\mu_{k+1}-\mu_{k}\right)\right)_{k \in \mathbb{Z}}$ is R-bounded. From [2, Theorem 1.3], we deduce that $\left(\mu_{k}\right)_{k \in \mathbb{Z}}$ is an $L^{p}$-multiplier. Then $M_{k}=\frac{-1}{1-a_{k}} \mu_{k}$ is also an $L^{p}$-multiplier by Lemma 3.4 and Remark 2.2. The proof is complete.

Now, we consider the maximal regularity for the problem $\left(P_{5}\right)$ on periodic Besov spaces $B_{p, q}^{s}(\mathbb{T}, X)$, where $1 \leq p, q \leq \infty, s>0$. By [3], if $X$ is an arbitrary Banach space, then the Marcinkiewicz condition of order 2 , that is,

$$
\sup _{k \in \mathbb{Z}}\left(\left\|M_{k}\right\|+\left\|k\left(M_{k+1}-M_{k}\right)\right\|+\left\|k^{2}\left(M_{k+1}-2 M_{k}+M_{k-1}\right)\right\|\right)<\infty,
$$

is sufficient for the sequence $\left(M_{k}\right)_{k \in \mathbb{Z}}$ to be a $B_{p, q}^{s}$-multiplier whenever $1 \leq$ $p, q \leq \infty$ and $s \in \mathbb{R}$. So for the maximal regularity of the problem $\left(P_{5}\right)$ in $B_{p, q}^{s}(\mathbb{T}, X)$, we must impose the stronger assumption $(\mathbf{H 2})$ on $a, b \in L^{1}\left(\mathbb{R}_{+}\right)$.

Theorem 3.6. Let $X$ be a Banach space and let $A$ be a closed linear operator in $X$. Assume that $a, b \in L^{1}\left(\mathbb{R}_{+}\right)$satisfy $(\mathbf{H 2})$ and a satisfies $(\mathbf{H 1} \mathbf{b})$. Then the following statements are equivalent:

(i) The problem $\left(P_{5}\right)$ has $B_{p, q}^{s}$-maximal regularity for some (equivalently, all $) 1 \leq p, q \leq \infty$ and $s>0$.

(ii) $\left(d_{k}\right)_{k \in \mathbb{Z}} \subset \varrho(A)$ and $\left(M_{k}\right)_{k \in \mathbb{Z}}$ is bounded.

Proof. Since $a, b \in L^{1}\left(\mathbb{R}_{+}\right)$satisfy (H2) and $a$ satisfies $(\mathbf{H 1} \mathbf{b})$, we see that $a$ satisfies (H0) and $b$ satisfies (H0a) by Lemma 3.4. Thus Theorem 2.4 is applicable in the case $\Gamma(\mathbb{T}, X)=B_{p, q}^{s}(\mathbb{T}, X)$ when $1 \leq p, q \leq \infty$ and $s>0$.

(i) $\Rightarrow$ (ii): Assume that $\left(P_{5}\right)$ has $B_{p, q}^{s}$-maximal regularity for some $1 \leq$ $p, q \leq \infty$ and $s>0$. Then in view of Theorem 2.4, $\left(d_{k}\right)_{k \in \mathbb{Z}} \subset \varrho(A)$ and $\left(M_{k}\right)_{k \in \mathbb{Z}}$ is a $B_{p, q}^{s}$-multiplier. Hence $\left(M_{k}\right)_{k \in \mathbb{Z}}$ must be bounded [3, Theorem 5.1].

(ii) $\Rightarrow$ (i): Let $1 \leq p, q \leq \infty$ and $s>0$ be fixed. To show that $\left(P_{5}\right)$ has $B_{p, q}^{s}$-maximal regularity, it suffices to prove that $\left(M_{k}\right)_{k \in \mathbb{Z}}$ is a $B_{p, q}^{s}$-multiplier by Theorem 2.4. We let $\mu_{k}=k^{2} R\left(d_{k}, A\right)$ for $k \in \mathbb{Z}$ and we first show that 
$\left(\mu_{k}\right)_{k \in \mathbb{Z}}$ is a $B_{p, q}^{s}$-multiplier. It is clear that (H2) implies (H1a). From the proof of Theorem 3.5, we know that $\left(\mu_{k}\right)_{k \in \mathbb{Z}}$ and $\left(k\left(\mu_{k+1}-\mu_{k}\right)\right)_{k \in \mathbb{Z}}$ are bounded. To show that $\left(\mu_{k}\right)_{k \in \mathbb{Z}}$ is a $B_{p, q}^{s}$-multiplier, we need only show that $\left(k^{2}\left(\mu_{k+1}-2 \mu_{k}+\mu_{k-1}\right)\right)_{k \in \mathbb{Z}}$ is bounded, by the Fourier multiplier theorem on periodic Besov spaces [3, Theorem 4.5]. For $k \in \mathbb{Z}$, we have

$$
\begin{aligned}
k^{2}\left(\mu_{k+1}-2 \mu_{k}+\mu_{k-1}\right)= & k^{4}\left[R\left(d_{k+1}, A\right)-2 R\left(d_{k}, A\right)+R\left(d_{k-1}, A\right)\right] \\
& +2 k^{3}\left[R\left(d_{k+1}, A\right)-R\left(d_{k-1}, A\right)\right] \\
& +k^{2}\left[R\left(d_{k+1}, A\right)+R\left(d_{k-1}, A\right)\right]=: I_{1}+I_{2}+I_{3} .
\end{aligned}
$$

For $I_{1}$, we have

$$
\begin{aligned}
I_{1}= & k^{4} R\left(d_{k}, A\right)\left[\left(d_{k}-d_{k+1}\right) R\left(d_{k+1}, A\right)-\left(d_{k-1}-d_{k}\right) R\left(d_{k-1}, A\right)\right] \\
= & \mu_{k} k^{2}\left[\left(d_{k}-d_{k+1}\right)\left(R\left(d_{k+1}, A\right)-R\left(d_{k-1}, A\right)\right)\right. \\
& \left.-\left(d_{k+1}-2 d_{k}+d_{k-1}\right) R\left(d_{k-1}, A\right)\right] \\
= & \frac{d_{k}-d_{k+1}}{k} \frac{d_{k-1}-d_{k+1}}{k} \mu_{k}\left(k^{2} R\left(d_{k+1}, A\right)\right)\left(k^{2} R\left(d_{k-1}, A\right)\right) \\
& -\mu_{k}\left(d_{k+1}-2 d_{k}+d_{k-1}\right)\left(k^{2} R\left(d_{k-1}, A\right)\right) .
\end{aligned}
$$

Since $\left(d_{k}-d_{k+1}\right) / k$ is bounded, so is

$$
\frac{d_{k-1}-d_{k+1}}{k}=\frac{d_{k-1}-d_{k}}{k}+\frac{d_{k}-d_{k+1}}{k} .
$$

The sequences $k^{2} R\left(d_{k-1}, A\right)=\frac{k^{2}}{(k-1)^{2}} \mu_{k-1}$ and $k^{2} R\left(d_{k+1}, A\right)=\frac{k^{2}}{(k+1)^{2}} \mu_{k+1}$ are bounded. To show that $I_{1}$ is bounded, it remains to consider $d_{k+1}-$ $2 d_{k}+d_{k-1}$. We have

$$
\begin{array}{rl}
d_{k+1}-2 d_{k}+d_{k-1} \\
=i & i k \alpha\left(\frac{1}{1-a_{k+1}}-\frac{2}{1-a_{k}}+\frac{1}{1-a_{k-1}}\right)+i \alpha\left(\frac{1}{1-a_{k+1}}-\frac{1}{1-a_{k-1}}\right) \\
+ & i\left(\frac{(k+1) b_{k+1}}{1-a_{k+1}}-\frac{2 k b_{k}}{1-a_{k}}+\frac{(k-1) b_{k-1}}{1-a_{k-1}}\right) \\
- & k^{2}\left(\frac{1}{1-a_{k+1}}-\frac{2}{1-a_{k}}+\frac{1}{1-a_{k-1}}\right) \\
& -\frac{2+2 k\left(a_{k+1}-a_{k-1}\right)-a_{k+1}-a_{k-1}}{\left(1-a_{k+1}\right)\left(1-a_{k-1}\right)} .
\end{array}
$$

Each term in the above expression is bounded by the assumption on $a, b \in$ $L^{1}\left(\mathbb{R}_{+}\right)$. We have shown that $I_{1}$ is bounded.

To estimate $I_{2}$ and $I_{3}$, we have

$$
\begin{aligned}
& I_{2}=\frac{2\left(d_{k-1}-d_{k+1}\right)}{k}\left(k^{2} R\left(d_{k+1}, A\right)\right)\left(k^{2} R\left(d_{k-1}, A\right)\right), \\
& I_{3}=k^{2} R\left(d_{k+1}, A\right)+k^{2} R\left(d_{k-1}, A\right) .
\end{aligned}
$$


Thus the boundedness of $I_{2}$ and $I_{3}$ follows easily from the boundedness of $\left(d_{k-1}-d_{k+1}\right) / k, k^{2} R\left(d_{k+1}, A\right)$ and $k^{2} R\left(d_{k-1}, A\right)$. We have shown that $\left(\mu_{k}\right)_{k \in \mathbb{Z}}$ satisfies the Marcinkiewicz condition of order 2. Therefore it is a $B_{p, q}^{s}$-multiplier [3, Theorem 4.5]. By the assumption on $a$ and Lemma 3.4, $\left(\left(1-a_{k}\right)^{-1}\right)_{k \in \mathbb{Z}}$ is also a $B_{p, q}^{s}$-multiplier. Therefore $\left(M_{k}\right)_{k \in \mathbb{Z}}$ is a $B_{p, q}^{s}$-multiplier by Remark 2.2. The proof is complete.

If the underlying Banach space $X$ has a non-trivial Fourier type and $1 \leq p, q \leq \infty, s \in \mathbb{R}$, then the Marcinkiewicz condition of order 1 , that is,

$$
\sup _{k}\left(\left\|M_{k}\right\|+\left\|k\left(M_{k+1}-M_{k}\right)\right\|\right)<\infty,
$$

is already sufficient for $\left(M_{k}\right)_{k \in \mathbb{Z}}$ to be a $B_{p, q}^{s}$-multiplier [3, Theorem 4.5]. From this fact and the proof of Theorem 3.5, we easily deduce the following result on the $B_{p, q}^{s}$-maximal regularity of $\left(P_{5}\right)$ under a weaker condition on $a, b$ when $X$ has a non-trivial Fourier type.

TheOREM 3.7. Let $X$ be a Banach space with non-trivial Fourier type. Assume that $a, b \in L^{1}\left(\mathbb{R}_{+}\right)$satisfy $(\mathbf{H 1})$ and $(\mathbf{H 1 a})$, respectively. Then for $1 \leq p, q \leq \infty$ and $s>0$, the following statements are equivalent:

(i) The problem $\left(P_{5}\right)$ has $B_{p, q}^{s}$-maximal regularity for some (equivalently, all) $1 \leq p, q \leq \infty, s>0$.

(ii) $\left(d_{k}\right)_{k \in \mathbb{Z}} \subset \varrho(A)$ and $\left(M_{k}\right)_{k \in \mathbb{Z}}$ is bounded.

Periodic Hölder continuous function spaces are a particular case of $B_{p, q}^{s}(\mathbb{T}, X)$. From $[3$, Theorem 3.1], we have

$$
B_{\infty, \infty}^{\alpha}(\mathbb{T}, X)=C_{\text {per }}^{\alpha}(\mathbb{T}, X) \quad \text { whenever } 0<\alpha<1,
$$

where $C_{\mathrm{per}}^{\alpha}(\mathbb{T}, X)$ is the space of all $X$-valued functions $f$ defined on $\mathbb{T}$ and such that $f(0)=f(2 \pi)$ and $\sup _{x \neq y}\|f(x)-f(y)\| /|x-y|^{\alpha}$ is finite. Moreover, the norm

$$
\|u\|_{C_{\mathrm{per}}^{\alpha}}:=\max _{t \in \mathbb{T}}\|u(t)\|+\sup _{x \neq y} \frac{\|f(x)-f(y)\|}{|x-y|^{\alpha}}
$$

on $C_{\mathrm{per}}^{\alpha}(\mathbb{T}, X)$ is an equivalent norm of $B_{\infty, \infty}^{\alpha}(\mathbb{T}, X)$. Thus Theorems 3.6 and 3.7 have the following corollary, where for $0<\alpha<1$ we say that

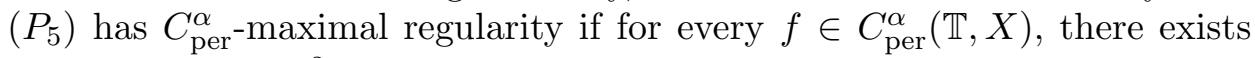
a unique $u \in C_{\mathrm{per}}^{\alpha+2}(\mathbb{T}, X)$ such that $u(t) \in D(A)$ and the equation of $\left(P_{5}\right)$ holds for all $t \in[0,2 \pi]$, and $u^{\prime \prime}, u^{\prime}, A u, a * A u, \frac{d}{d t}(b \dot{*} u) \in C_{\text {per }}^{\alpha}(\mathbb{T}, X)$.

Corollary 3.8. Let $X$ be a Banach space and let $a, b \in L^{1}\left(\mathbb{R}_{+}\right)$. Then:

1. If $a, b \in L^{1}\left(\mathbb{R}_{+}\right)$satisfy $(\mathbf{H 2})$ and a satisfies $(\mathbf{H 1} \mathbf{b})$, then the problem $\left(P_{5}\right)$ has $C_{\mathrm{per}}^{\alpha}$-maximal regularity for some (equivalently, all) $0<\alpha<1$ if and only if $\left(d_{k}\right)_{k \in \mathbb{Z}} \subset \varrho(A)$ and $\left(M_{k}\right)_{k \in \mathbb{Z}}$ is bounded. 
2. If $X$ has a non-trivial Fourier type and if a satisfies (H1) and $b$ satisfies $(\mathbf{H 1 a})$, then the problem $\left(P_{5}\right)$ has $C_{\mathrm{per}}^{\alpha}$-maximal regularity for some (equivalently, all) $0<\alpha<1$ if and only if $\left(d_{k}\right)_{k \in \mathbb{Z}} \subset \varrho(A)$ and $\left(M_{k}\right)_{k \in \mathbb{Z}}$ is bounded.

Let $M=\left(M_{k}\right)_{k \in \mathbb{Z}} \subset \mathcal{L}(X, Y)$. We say that $M$ satisfies the Marcinkiewicz condition of order 3 if $M$ satisfies the Marcinkiewicz condition of order 2 and

$$
\sup _{k}\left\|k^{3}\left(M_{k+1}-3 M_{k}+3 M_{k-1}-M_{k-2}\right)\right\|<\infty
$$

(see [6]). Next, we prove maximal regularity of $\left(P_{5}\right)$ on periodic Triebel spaces $F_{p, q}^{s}(\mathbb{T}, X)$ when $1 \leq p<\infty, 1 \leq q \leq \infty, s>0$. We need the stronger condition (H3) on $a, b \in L^{1}\left(\mathbb{R}_{+}\right)$because the Marcinkiewicz condition of order 3 is needed in the $F_{p, q}^{s}$-multiplier case [6, Theorem 3.2].

Theorem 3.9. Let $X$ be a Banach space. Assume that $a, b \in L^{1}\left(\mathbb{R}_{+}\right)$ satisfy $(\mathbf{H 3})$ and a satisfies $(\mathbf{H 1} \mathbf{b})$. Then the following assertions are equivalent:

(i) The problem $\left(P_{5}\right)$ has $F_{p, q}^{s}$-maximal regularity for some (equivalently, all) $1 \leq p<\infty, 1 \leq q \leq \infty$ and $s>0$.

(ii) $\left(d_{k}\right)_{k \in \mathbb{Z}} \subset \varrho(A)$ and $\left(M_{k}\right)_{k \in \mathbb{Z}}$ is bounded.

Proof. Since $a, b \in L^{1}\left(\mathbb{R}_{+}\right)$satisfy $(\mathbf{H 3})$ and $a$ satisfies $(\mathbf{H 1 b})$, we infer that $a$ satisfies (H0) and $b$ satisfies (H0a) by Lemma 3.4. Thus Theorem 2.4 is applicable in the case $\Gamma(\mathbb{T}, X)=F_{p, q}^{s}(\mathbb{T}, X)$ when $1 \leq p<\infty, 1 \leq q \leq \infty$ and $s>0$.

(i) $\Rightarrow$ (ii): Assume that $\left(P_{5}\right)$ has $F_{p, q}^{s}$-maximal regularity for some $1 \leq$ $p<\infty, 1 \leq q \leq \infty$ and $s>0$. Then $\left(d_{k}\right)_{k \in \mathbb{Z}} \subset \varrho(A)$ and $\left(M_{k}\right)_{k \in \mathbb{Z}}$ is an $F_{p, q}^{s}$-multiplier by Theorem 2.4 . Hence $\left(M_{k}\right)_{k \in \mathbb{Z}}$ must be bounded [6, Theorem 4.1].

(ii) $\Rightarrow$ (i): Let $1 \leq p<\infty, 1 \leq q \leq \infty$ and $s>0$ be fixed. To show that $\left(P_{5}\right)$ has $F_{p, q}^{s}$-maximal regularity, it suffices to prove that $\left(M_{k}\right)_{k \in \mathbb{Z}}$ is an $F_{p, q^{-}}^{s}$ multiplier by Theorem 2.4. We let $\mu_{k}=k^{2} R\left(d_{k}, A\right)$ for $k \in \mathbb{Z}$ and we first show that $\left(\mu_{k}\right)_{k \in \mathbb{Z}}$ is an $F_{p, q}^{s}$-multiplier. It is clear that (H3) implies (H2). Thus the boundedness of $\mu_{k}, k\left(\mu_{k+1}-\mu_{k}\right)$ and $k^{2}\left(\mu_{k+1}-2 \mu_{k}+\mu_{k-1}\right)$ follows from the proofs of Theorems 3.5 and 3.6. It remains to show that $k^{3}\left(\mu_{k+1}-\right.$ $\left.3 \mu_{k}+3 \mu_{k-1}-\mu_{k-2}\right)$ is bounded. We have

$$
\begin{aligned}
& k^{3}\left(\mu_{k+1}-3 \mu_{k}+3 \mu_{k-1}-\mu_{k-2}\right) \\
& =k^{5}\left[R\left(d_{k+1}, A\right)-3 R\left(d_{k}, A\right)+3 R\left(d_{k-1}, A\right)-R\left(d_{k-2}, A\right)\right] \\
& \quad+2 k^{4}\left[R\left(d_{k+1}, A\right)-3 R\left(d_{k-1}, A\right)+2 R\left(d_{k-2}, A\right)\right] \\
& \quad+k^{3}\left[R\left(d_{k+1}, A\right)+3 R\left(d_{k-1}, A\right)-4 R\left(d_{k-2}, A\right)\right]=: J_{1}+J_{2}+J_{3} .
\end{aligned}
$$


Now,

$$
\begin{aligned}
& J_{1}=-k\left(d_{k+1}-3 d_{k}+3 d_{k-1}-d_{k-2}\right) \frac{k^{2} \mu_{k} \mu_{k-1}}{(k-1)^{2}} \\
& +\frac{d_{k+1}-d_{k-2}}{k}\left(d_{k+1}-2 d_{k}+d_{k-1}\right) \frac{k^{4} \mu_{k-1} \mu_{k} \mu_{k+1}}{\left(k^{2}-1\right)^{2}} \\
& +\frac{d_{k+1}-d_{k-2}}{k}\left(d_{k}-2 d_{k-1}+d_{k-2}\right) \frac{k^{4} \mu_{k-2} \mu_{k} \mu_{k+1}}{\left(k^{2}-k-2\right)^{2}} \\
& +2 \frac{d_{k+1}-d_{k-2}}{k} \frac{d_{k}-d_{k-1}}{k} \frac{d_{k-2}-d_{k-1}}{k} \frac{k^{6} \mu_{k-2} \mu_{k-1} \mu_{k} \mu_{k+1}}{\left(k^{3}-2 k^{2}-k+2\right)^{2}} \text {, } \\
& J_{2}=-2\left(d_{k+1}-2 d_{k}+d_{k-1}\right) \frac{k^{2} \mu_{k-1} \mu_{k}}{(k-1)^{2}} \\
& +2 \frac{d_{k}-d_{k+1}}{k} \frac{d_{k-1}-d_{k+1}}{k} \frac{k^{4} \mu_{k-1} \mu_{k} \mu_{k+1}}{\left(k^{2}-1\right)^{2}} \\
& +4 \frac{d_{k-1}-d_{k}}{k} \frac{d_{k-2}-d_{k}}{k} \frac{k^{4} \mu_{k-2} \mu_{k-1} \mu_{k}}{\left(k^{2}-3 k+2\right)^{2}} \\
& -4\left(d_{k}-2 d_{k-1}+d_{k-2}\right) \frac{k^{4} \mu_{k-2} \mu_{k-1}}{\left(k^{2}-3 k+2\right)^{2}}, \\
& J_{3}=\frac{d_{k-2}-d_{k+1}}{k} \frac{k^{4} \mu_{k-2} \mu_{k+1}}{\left(k^{2}-k-2\right)^{2}} \\
& +\frac{3\left(d_{k-2}-d_{k-1}\right)}{k} \frac{k^{4} \mu_{k-2} \mu_{k-1}}{\left(k^{2}-3 k+2\right)^{2}} .
\end{aligned}
$$

The boundedness of $\mu_{k},\left(d_{k}-d_{k+1}\right) / k$ and $d_{k+1}-2 d_{k}+d_{k-1}$ follows from the proof of Theorem 3.6. To show that $J_{1}, J_{2}$ and $J_{3}$ are bounded, it suffices to show that $k\left(d_{k+1}-3 d_{k}+3 d_{k-1}-d_{k-2}\right)$ is bounded. We have

$$
\begin{aligned}
k\left(d_{k+1}-3 d_{k}+3 d_{k-1}-d_{k-2}\right) & \\
= & i k^{2} \alpha\left(\frac{1}{1-a_{k+1}}-\frac{3}{1-a_{k}}+\frac{3}{1-a_{k-1}}-\frac{1}{1-a_{k-2}}\right) \\
& +i k \alpha\left(\frac{1}{1-a_{k+1}}-\frac{3}{1-a_{k-1}}+\frac{2}{1-a_{k-2}}\right) \\
& +i k\left(\frac{b_{k+1}}{1-a_{k+1}}-\frac{3 b_{k-1}}{1-a_{k-1}}+\frac{2 b_{k-2}}{1-a_{k-2}}\right) \\
& +i k^{2}\left[\left(\frac{b_{k+1}}{1-a_{k+1}}-\frac{2 b_{k}}{1-a_{k}}+\frac{b_{k-1}}{1-a_{k-1}}\right)\right.
\end{aligned}
$$




$$
\begin{aligned}
& \left.-\left(\frac{b_{k}}{1-a_{k}}-\frac{2 b_{k-1}}{1-a_{k-1}}+\frac{b_{k-2}}{1-a_{k-2}}\right)\right] \\
& -k^{3}\left[\frac{1}{1-a_{k+1}}-\frac{3}{1-a_{k}}+\frac{3}{1-a_{k-1}}-\frac{1}{1-a_{k-2}}\right] \\
& -k\left[\frac{2 k+1}{1-a_{k+1}}+\frac{3(-2 k+1)}{1-a_{k-1}}-\frac{-4 k+4}{1-a_{k-2}}\right]
\end{aligned}
$$

The boundedness of the first and fifth brackets follows from the proof of [5, Proposition 3.4]. The fourth bracket is bounded by the proof of [11, Theorem 3.12]. We also have

$$
\begin{aligned}
i k \alpha & \left(\frac{1}{1-a_{k+1}}-\frac{3}{1-a_{k-1}}+\frac{2}{1-a_{k-2}}\right) \\
\quad & i \alpha \frac{k\left(a_{k+1}+2 a_{k-2}-3 a_{k-1}\right)+k a_{k-1} a_{k-2}-3 k a_{k+1} a_{k-2}+2 k a_{k+1} a_{k-1}}{\left(1-a_{k+1}\right)\left(1-a_{k-1}\right)\left(1-a_{k-2}\right)}
\end{aligned}
$$

and

$$
\begin{array}{r}
k\left[\frac{2 k+1}{1-a_{k+1}}+\frac{3(-2 k+1)}{1-a_{k-1}}-\frac{-4 k+4}{1-a_{k-2}}\right] \\
=\frac{J}{\left(1-a_{k+1}\right)\left(1-a_{k-1}\right)\left(1-a_{k-2}\right)}
\end{array}
$$

where

$$
\begin{aligned}
J= & 2 k^{2}\left(a_{k+1}-2 a_{k}+a_{k-1}\right)+4 k^{2}\left(a_{k}-2 a_{k-1}+a_{k-2}\right) \\
& +\left(3 k a_{k-1}-4 k a_{k-2}+k a_{k+1}\right)+(2 k+1) k a_{k-1} a_{k-2} \\
& +(-6 k+3) k a_{k+1} a_{k-2}+(4 k-4) k a_{k+1} a_{k-1} .
\end{aligned}
$$

We have shown that $k\left(d_{k+1}-3 d_{k}+3 d_{k-1}-d_{k-2}\right)$ is bounded by the assumption on $a \in L^{1}\left(\mathbb{R}_{+}\right)$. We deduce that $\left(\mu_{k}\right)_{k \in \mathbb{Z}}$ is an $F_{p, q}^{s}$-multiplier by [6, Theorem 3.2]. From the assumptions on $a \in L^{1}\left(\mathbb{R}_{+}\right)$and Lemma 3.4, $\left(\left(1-a_{k}\right)^{-1}\right)_{k \in \mathbb{Z}}$ is also an $F_{p, q}^{s}$-multiplier. From Remark 2.2, we deduce that $M_{k}=\frac{-1}{1-a_{k}} \mu_{k}$ is an $F_{p, q}^{s}$-multiplier. The proof is finished.

Remark 3.10. When $1<p<\infty, 1<q \leq \infty$ and $s \in \mathbb{R}$, the Marcinkiewicz condition of order 2 is already sufficient for a sequence $\left(M_{k}\right)_{k \in \mathbb{Z}} \subset$ $\mathcal{L}(X)$ to be an $F_{p, q}^{s}$-multiplier [6, Theorem 3.2]. This fact together with the proof of Theorem 3.6 implies that if $a, b \in L^{1}\left(\mathbb{R}_{+}\right)$satisfy $(\mathbf{H 2})$, and $a$ satisfies $(\mathbf{H} 1 \mathbf{b})$, then the problem $\left(P_{5}\right)$ has $F_{p, q}^{s}$-maximal regularity for some (equivalently, all) $1<p<\infty, 1<q \leq \infty$ and $s>0$ if and only if $\left(d_{k}\right)_{k \in \mathbb{Z}} \subset \varrho(A)$ and $\left(M_{k}\right)_{k \in \mathbb{Z}}$ is bounded.

Acknowledgements. The authors thank the referee for many useful comments and valuable suggestions. 


\section{References}

[1] W. Arendt, C. Batty and S. Q. Bu, Fourier multipliers for Hölder continuous functions and maximal regularity, Studia Math. 160 (2004), 23-51.

[2] W. Arendt and S. Q. Bu, The operator-valued Marcinkiewicz multiplier theorem and maximal regularity, Math. Z. 240 (2002), 311-343.

[3] -, 一, Operator-valued Fourier multipliers on periodic Besov spaces and applications, Proc. Edinburgh Math. Soc. 47 (2004), 15-33.

[4] J. Bourgain, Vector-valued singular integrals and the $H^{1}-B M O$ duality, in: Probability Theory and Harmonic Analysis (Cleveland, OH, 1983), Monogr. Textbooks Pure Appl. Math. 98, Dekker, New York, 1988, 1-19.

[5] S. Q. Bu and Y. Fang, Maximal regularity for integro-differential equations in periodic Triebel-Lizorkin spaces, Taiwanese J. Math., to appear.

[6] S. Q. Bu and J. M. Kim, Operator-valued Fourier multipliers on periodic Triebel spaces, Acta Math. Sinica (English Ser.) (5) 21 (2005), 1049-1056.

[7] R. Chill and S. Srivastava, $L^{p}$-maximal regularity for second order Cauchy problems, Math. Z. 251 (2005), 751-781.

[8] Ph. Clément and G. Da Prato, Existence and regularity results for an integral equation with infinite delay in a Banach space, Integral Equations Operator Theory 11 (1988), 480-550.

[9] G. Da Prato and A. Lunardi, Solvability on the real line of a class of linear Volterra integro-differential equations of parabolic type, Ann. Mat. Pura Appl. (4) 150 (1988), $67-117$.

[10] V. Keyantuo and C. Lizama, Fourier multipliers and integro-differential equations in Banach spaces, J. London Math. Soc. (2) 69 (2004), 737-750.

[11] - - - Maximal regularity for a class of integro-differential equations with infinite delay in Banach spaces, Studia Math. 168 (2005), 25-50.

[12] A. Lunardi, The heat equation with fading memory, SIAM J. Math. Anal. 21 (1990), $1213-1224$.

[13] A. Pietsch and J. Wenzel, Orthonormal Systems and Banach Space Geometry, Encyclopedia Math. Appl. 70, Cambridge Univ. Press, 1998.

[14] L. Weis, Operator-valued Fourier multipliers and maximal $L^{p}$-regularity, Math. Ann. 319 (2001), 735-758.

[15] - A new approach to maximal $L^{p}$-regularity, in: Evolution Equations and Their Applications in Physical and Life Sciences (Bad Herrenalb, 1998), Lecture Notes in Pure Appl. Math. 215, Dekker, New York, 2001, 195-214.

Department of Mathematical Science

University of Tsinghua

Beijing 100084, China

E-mail: sbu@math.tsinghua.edu.cn

fangy04@mails.tsinghua.edu.cn

Received November 7, 2005

Revised version October 24, 2007 